\title{
New source for ultracold neutrons at the Institut Laue-Langevin
}

\author{
F. M. Piegsa, ${ }^{1,2,{ }^{*}}$ M. Fertl, ${ }^{1,3, \dagger}$ S. N. Ivanov,${ }^{1}$ M. Kreuz, ${ }^{1}$ K. K. H. Leung,,${ }^{1,}$ P. Schmidt-Wellenburg, ${ }^{1,3}$ \\ T. Soldner, ${ }^{1}$ and O. Zimmer ${ }^{1, \S}$ \\ ${ }^{1}$ Institut Laue-Langevin, BP 156, F-38042 Grenoble, France \\ ${ }^{2}$ ETH Zürich, Institute for Particle Physics, CH-8093 Zürich, Switzerland \\ ${ }^{3}$ Paul Scherrer Institute, $\mathrm{CH}-5232$ Villigen PSI, Switzerland
}

(Received 7 May 2013; revised manuscript received 28 February 2014; published 23 July 2014)

\begin{abstract}
A new intense superthermal source for ultracold neutrons (UCN) was installed at a dedicated beam line at the Institut Laue-Langevin. Incident neutrons with a wavelength of $0.89 \mathrm{~nm}$ are converted to UCN in a 5-liter volume filled with superfluid ${ }^{4} \mathrm{He}$ at a temperature of about $0.7 \mathrm{~K}$. The UCN can be extracted to room temperature experiments. We present the cryogenic setup of the source, a characterization of the cold neutron beam, and UCN production measurements, where a UCN density in the production volume of at least 55 per $\mathrm{cm}^{3}$ was determined.
\end{abstract}

DOI: 10.1103/PhysRevC.90.015501

PACS number(s): 29.25.Dz, 07.20.Mc, 67.85.-d, 78.70.Nx

\section{INTRODUCTION}

The neutron offers access to many different physical phenomena. Its static and decay properties as well as its interactions are studied at research reactors and spallation sources. High precision experiments have impact on particle physics and cosmology and also serve for sensitive searches for new physics, complementary to work done at high-energy accelerators [1-3]. For several important investigations ultracold neutrons (UCN) have proven to be particularly useful. The defining property of UCN is total reflection under any angle of incidence by virtue of the neutron optical potential. As a result one can confine them in "neutron bottles" made of suitable materials with small cross sections for neutron absorption and trap depths of up to about $300 \mathrm{neV}$. Good neutron bottles provide storage time constants of many hundreds of seconds [4].

Current fields of research with UCN include the search for a nonvanishing neutron electric dipole moment [5-13], searches for "mirror matter" as a viable candidate for dark matter [14,15], sensitive tests of Lorentz invariance [16,17], searches for new fundamental forces mediated by axionlike particles [18-20], measurements of the neutron lifetime [21$25]$, and angular correlation measurements in neutron $\beta$ decay [26]. UCN are also useful for investigations of quantum mechanical phenomena, e.g., quantum states of the neutron in the earth's gravitational field [27-29]. The new source described here will provide UCN for the GRANIT experiment, a gravitational quantum level experiment, which is currently being set up at the Institut Laue-Langevin (ILL) in Grenoble, France [30,31].

The development of new sources has become strongly motivated by counting statistical limitations apparent in many

\footnotetext{
*florian.piegsa@phys.ethz.ch

†Present address: Department of Physics and Center for Experimental Nuclear Physics and Astrophysics, University of Washington, Seattle, WA 98195, USA.

${ }_{\ddagger}^{\ddagger}$ Present address: Physics Department, North Carolina State University, Raleigh, NC 27695, USA.

$\S_{\text {zimmer@ill.fr }}$
}

of the aforementioned studies, which mostly were performed at the UCN source PF2 at ILL, consisting of a neutron turbine coupled to a liquid deuterium moderator [32]. To overcome these limitations several laboratories have embarked in developing UCN sources of a "next generation" [33-41], all based on the "superthermal" UCN production scheme proposed by Golub and Pendlebury in 1975 [42]. In this concept neutrons incident on a cold converter made of solid deuterium or superfluid ${ }^{4} \mathrm{He}$ lose almost their entire energy in single scattering events, producing elementary excitations (phonons) in the converter medium which are cooled away by a refrigerator. At low temperatures the probability for upscattering of UCN back to higher energies is suppressed by the Boltzmann factor. In solid deuterium the survival time for free neutrons is only a fraction of a second. For competitive UCN densities a $\mathrm{SD}_{2}$ converter therefore needs to be implemented "in-pile" close to the core of a reactor or near a spallation target. On the other hand, ${ }^{4} \mathrm{He}$ has no cross section for neutron absorption so that a viable concept for production of competitive UCN densities places the converter at the end of a neutron guide [43]. No extraordinary cooling power is required there so that the source can be designed as a relocatable apparatus easy to access for maintenance and trouble-shooting. UCN storage time constants in ${ }^{4} \mathrm{He}$ are ultimately limited only by the neutron beta decay lifetime of about $880 \mathrm{~s}[44,45]$. Therefore, albeit a neutron guide delivers a much lower flux than available in-pile, UCN may be accumulated to high density in a converter with reflective walls. The kinematics defined by the dispersion relations of helium and the free neutron enables down-scatter of cold neutrons with an energy around $1.0 \mathrm{meV}$ (corresponding to a neutron wavelength of $0.89 \mathrm{~nm}$ ) to ultracold energies via emission of a single phonon. To a lower extent, also multiphonon processes contribute to the integral UCN production rate for a wide range of incident neutron energies [46-48].

While an attempt to extract accumulated UCN horizontally from a superfluid helium converter was hampered by large losses [49], vertical UCN extraction through a cold mechanical valve for UCN does not require any windows in the UCN guide connecting the converter with an experiment at room temperature. Already some years ago the concept was shown 


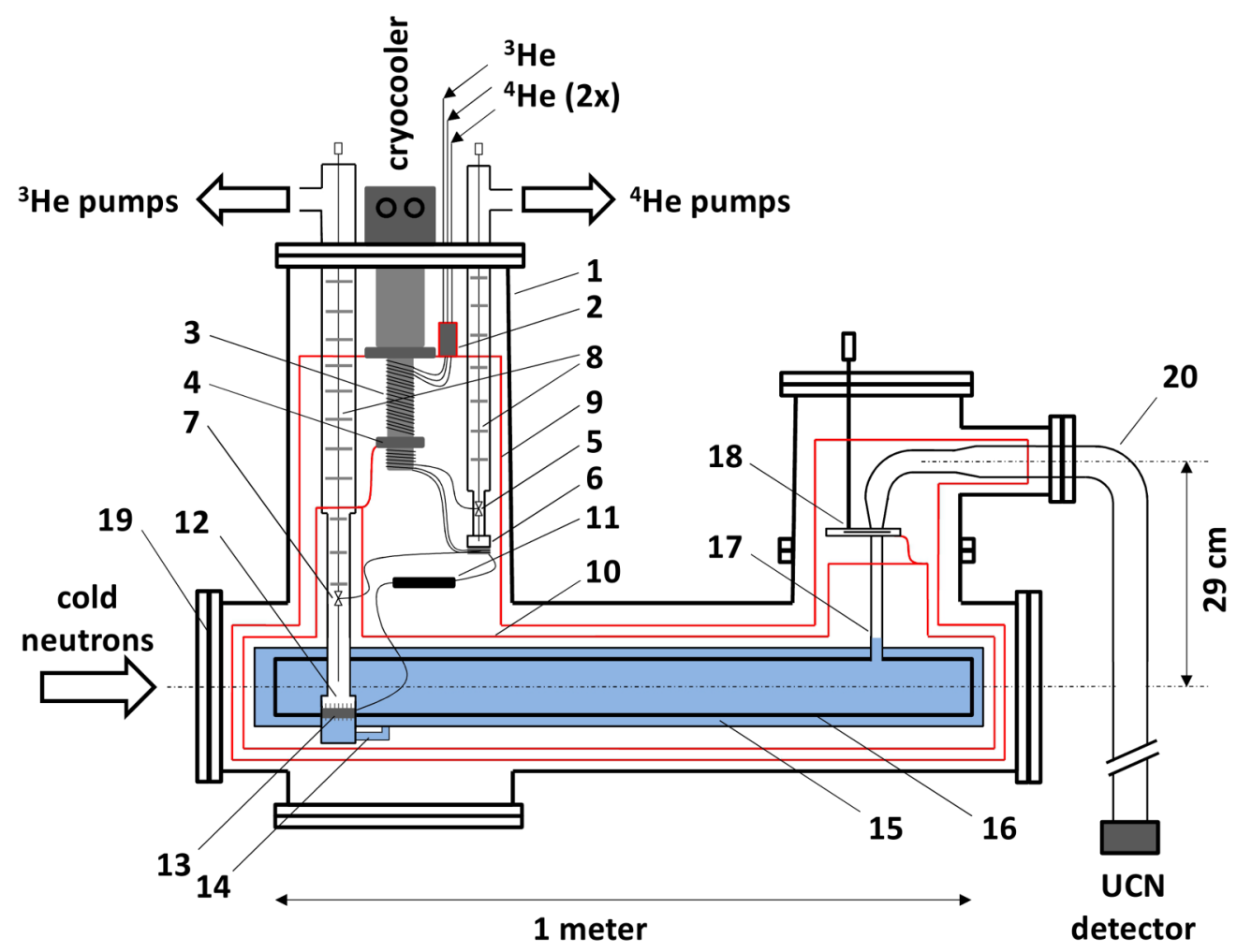

FIG. 1. (Color online) Schematic drawing of the cryostat of the UCN source: (1) isolation vacuum vessel, (2) cold traps and heat exchangers for incoming helium gas thermally anchored to the first stage of the cryocooler at about $45 \mathrm{~K}$, (3) regenerator heat exchangers, (4) second stage of the cryocooler with heat exchangers, (5) cold needle valve of the ${ }^{4} \mathrm{He}$ circuit, (6) $1-\mathrm{K}{ }^{4} \mathrm{He}$ evaporation pot, (7) cold needle valve of the ${ }^{3} \mathrm{He}$ circuit, (8) thermal baffles, (9) outer heat shield attached to the first stage of the cryocooler, (10) inner heat shield attached to the second stage of the cryocooler, (11) superleak filter, (12) ${ }^{3} \mathrm{He}$ evaporation pot, (13) copper heat exchanger, (14) "U"'-shaped connection pipe, (15) aluminum converter vessel filled with superfluid ${ }^{4} \mathrm{He}$, (16) UCN storage and production volume, (17) vertical stainless steel UCN guide, (18) UCN shutter valve thermally anchored to the inner heat shield, (19) thin aluminum cold neutron beam window, and (20) stainless steel UCN guide at room temperature.

to solve the problem, using a prototype apparatus installed at a neutron beam at the Munich research reactor FRM II [50,51]. ${ }^{1}$ In continuation of that development with the goal to make the technique available for the GRANIT experiment, the apparatus was upgraded and implemented at the new beam line $\mathrm{H} 172 \mathrm{a}$ at ILL [31,52]. Results of a commissioning run at the end of 2010 were already published [53]. Here, we give a detailed description of the source, the measured characteristics of the beam, and present more results on UCN production obtained with this improved device.

\section{THE ULTRACOLD NEUTRON SOURCE}

The key component of the presented superthermal UCN source is the cryostat which cools a large aluminum converter vessel filled with approximately 7 liters of superfluid ${ }^{4} \mathrm{He}$

\footnotetext{
${ }^{1}$ Despite a difference of potential energy of about $102 \mathrm{neV}$ per meter rise of a neutron in the gravity field, a short vertical section of the well-polished UCN guide is no obstacle for extraction if it does not exceed about $20 \mathrm{~cm}$. This is from UCN getting boosted by the neutron optical potential of the superfluid ${ }^{4} \mathrm{He}$ when they leave through the surface of the bath.
}

to below $1 \mathrm{~K}$. The converter liquid is cooled by coupling it thermally to a pumped ${ }^{3} \mathrm{He}$ bath. In Fig. 1 a schematic drawing of the complete cryogenic system is depicted. All low temperature parts are enclosed in an isolation vacuum vessel ( $p_{\text {iso }}<10^{-6}$ mbar) and two thermal heat radiation shields produced from thin copper and aluminum sheets. Two separate closed cooling circuits using ${ }^{3} \mathrm{He}$ and ${ }^{4} \mathrm{He}$ are fed into the cryostat via two gas inlets. A third inlet serves to fill the converter vessel with ${ }^{4} \mathrm{He}$ from a gas cylinder. All helium gas is filtered by passing it through charcoal cold traps immersed in liquid nitrogen (located external to the cryostat and not shown in Fig. 1) to prevent blocking of capillaries inside the cryostat by gas impurities. A commercial Gifford-McMahon cryocooler (Sumitomo RDK-415E, $1.5 \mathrm{~W}$ at $4.2 \mathrm{~K}$ ) is used to precool and finally liquefy the gas via heat exchangers attached to the first and second stage of the cryocooler, and to the regenerator between the two stages [54]. For all temperature measurements calibrated Lakeshore Cernox thermometers with a typical sensor accuracy of $\pm 5 \mathrm{mK}$ were used. The ${ }^{4} \mathrm{He}$ circuit is operated at an absolute inlet pressure of about 5-6 bar employing a small membrane compressor (KNF - N145 AN 9E). Hence, the ${ }^{4} \mathrm{He}$ gas can be liquefied on the second stage of the cryocooler even at elevated temperatures in the range $4.5-5.0 \mathrm{~K}$ caused by the thermal load. The liquid 
${ }^{4} \mathrm{He}$ is then continuously transferred into the so-called $1-\mathrm{K}$ pot, which has a volume of about $40 \mathrm{~cm}^{3}$. The flow of liquid ${ }^{4} \mathrm{He}$ is regulated using a cold needle valve to approximately $30-40 \mathrm{~g} / \mathrm{h}$. Two $40 \mathrm{~m}^{3} / \mathrm{h}$ dry pumps (Alcatel ACP 40 ) are used in parallel to pump on the $1-\mathrm{K}$ pot achieving a temperature of 1.5-2.0 K. This temperature strongly depends on the flow rate of ${ }^{3} \mathrm{He}$, as the precooled ${ }^{3} \mathrm{He}$ gas coming from the second stage of the cryocooler is liquefied in a heat-exchanger capillary situated inside the $1-\mathrm{K}$ pot. The ${ }^{3} \mathrm{He}$ circuit is operated at a much lower absolute inlet pressure of about 0.8 bar. After being liquefied, the ${ }^{3} \mathrm{He}$ is continuously transferred into the ${ }^{3} \mathrm{He}$ pot. The flow of liquid ${ }^{3} \mathrm{He}$ is regulated by a cold needle valve to about $10-15 \mathrm{~g} / \mathrm{h}$. By pumping with two consecutive roots pumps (Pfeiffer, $2000 \mathrm{~m}^{3} / \mathrm{h}$ and $500 \mathrm{~m}^{3} / \mathrm{h}$ ) backed by a dry pre-pump (Alcatel ACP 40) a base temperature of $0.55 \mathrm{~K}$ is reached in the ${ }^{3} \mathrm{He}$ pot. The total amount of circulating ${ }^{3} \mathrm{He}$ is about $1.5 \mathrm{~g}$. The ${ }^{4} \mathrm{He}$ gas used to fill the converter vessel is also precooled and liquefied on the stages of the cryocooler and then further cooled below the superfluid transition temperature and filtered by passing a "superleak" thermally anchored to the 1-K pot [51]. This filtering is important to remove traces of the strongly neutron absorbing isotope ${ }^{3} \mathrm{He}$ from the liquid. Finally, the heat transfer between the ${ }^{3} \mathrm{He}$ pot and the converter vessel is achieved by a heat exchanger produced from copper of high purity (a quality normally used for single crystal neutron monochromator production), shown in Fig. 2. The imperfect heat transfer of the heat exchanger, from the Kapitza resistance, results in a temperature gradient of about $0.15 \mathrm{~K}$, which causes a slightly higher temperature of the superfluid ${ }^{4} \mathrm{He}$ in the converter of about $0.7 \mathrm{~K}$ (measured with a resistor thermometer attached on the outside of the thin-walled "U"-shaped copper pipe; item 14 in Fig. 1). A thermometer of the same type mounted at the opposite end on the outside of the converter vessel showed a higher temperature of $0.85 \mathrm{~K}$, which might be because of a worse thermal coupling of the thermometer to the liquid helium (the thermometer was attached at a position above the helium bath, where the aluminum of the vessel has a thickness of approximately $1 \mathrm{~cm}$ and is not necessarily in direct contact with the superfluid). To

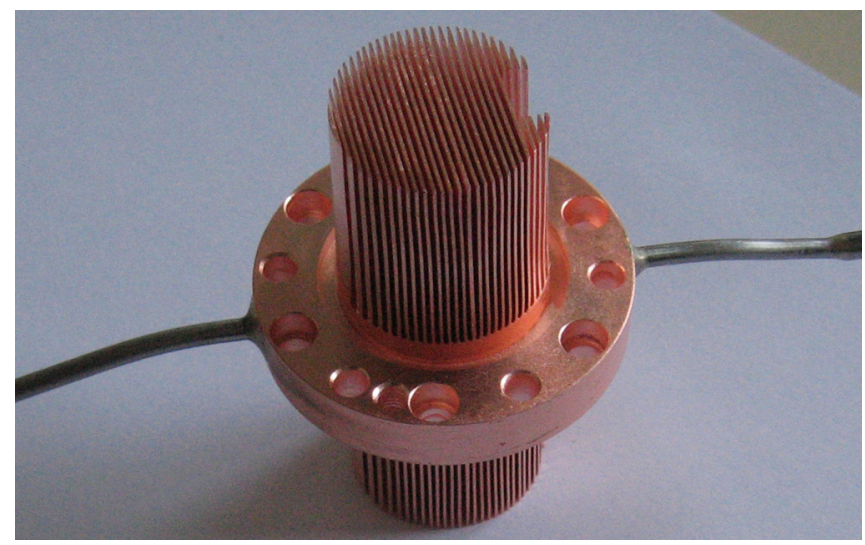

FIG. 2. (Color online) Copper heat exchanger between the ${ }^{3} \mathrm{He}$ pot and the superfluid ${ }^{4} \mathrm{He}$ bath in the converter vessel. The eroded fins (28-mm long and $0.5-\mathrm{mm}$ thick) result in a surface area of about $300 \mathrm{~cm}^{2}$ on each side.
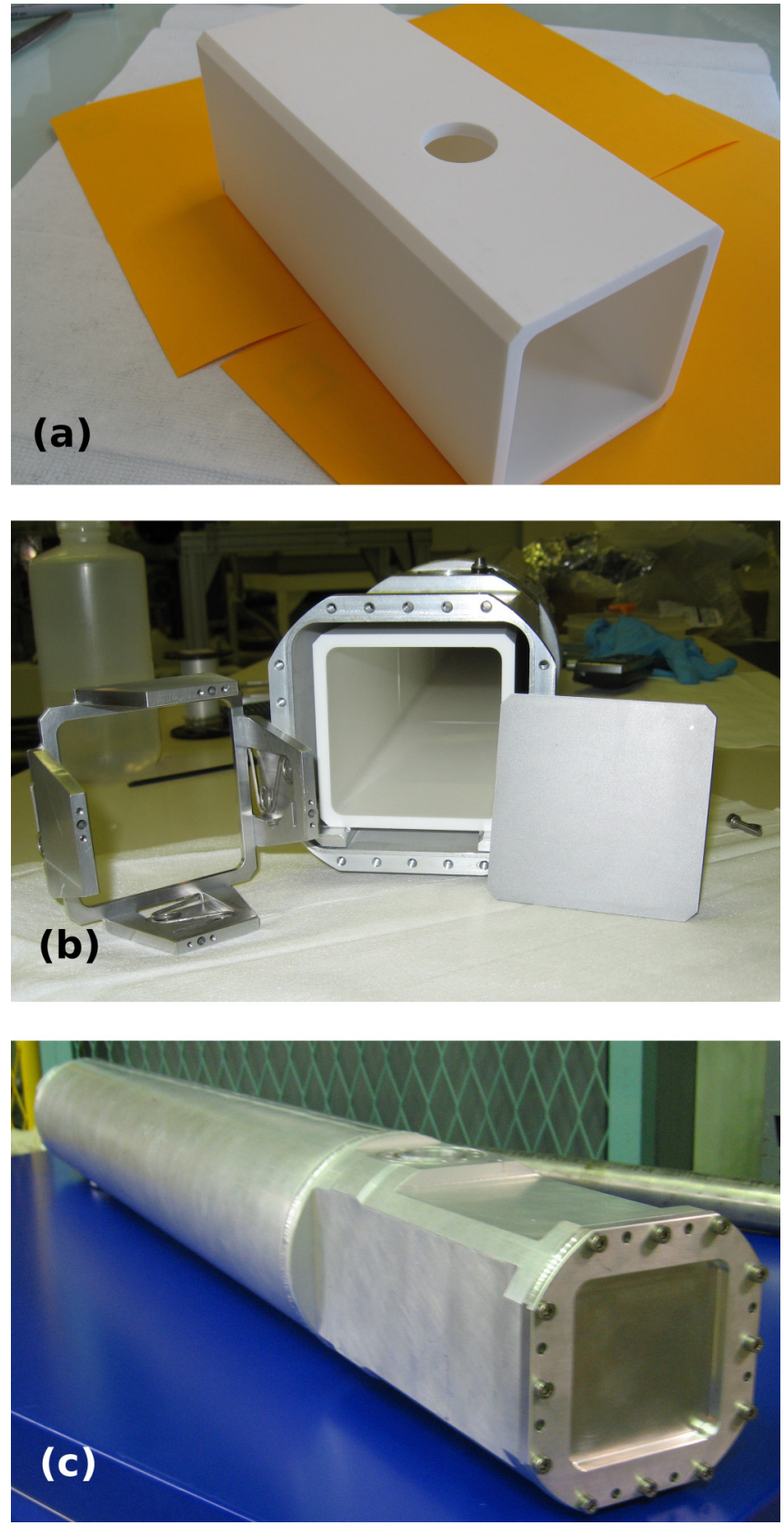

FIG. 3. (Color online) (a) One of the five 20-cm long $\mathrm{BeO}$ ceramic pieces forming the 5-liter UCN storage volume. Its inner cross section is $70 \times 70 \mathrm{~mm}^{2}$. The hole in the piece shown serves for extraction of the UCN via a stainless steel guide (see text). (b) Front view of the opened aluminum converter vessel with the installed white $\mathrm{BeO}$ ceramic pieces. The storage volume is closed off with two 1-mm thick beryllium sheets (right) serving as entrance and exit windows for the cold neutron beam. The spring mechanism used to press the ceramic parts together is presented on the left. (c) Closed converter vessel with an indium-sealed thin aluminum entrance window (approximate converter vessel length, $1 \mathrm{~m}$ ).

store the produced UCN a 5-liter UCN storage and production volume with the inner dimensions $70 \times 70 \times 1000 \mathrm{~mm}^{3}$ is placed inside the converter vessel. This volume is composed of five pieces of $\mathrm{BeO}$ ceramics and two 1-mm thick beryllium 
end caps (compare Fig. 3). Both materials have large neutron optical potentials (BeO: $261 \mathrm{neV}$ and $\mathrm{Be}: 252 \mathrm{neV}$ [4]) and low neutron absorption cross sections. To avoid UCN losses through potential gaps from thermal contraction, the set of $\mathrm{BeO}$ ceramics is pressed together by a spring mechanism.

For UCN extraction, a guide system is connected to a hole with diameter $24 \mathrm{~mm}$ in one of the $\mathrm{BeO}$ pieces. The entire $\mathrm{UCN}$ extraction guide system consisting of various tubing elements is made from polished stainless steel with a calculated neutron optical potential of $184 \mathrm{neV}$. The first, vertical section of the UCN extraction guide with inner diameter $23 \mathrm{~mm}$ is made to fit tightly into the hole in the $\mathrm{BeO}$ ceramics. Thirty millimeters below the end of this 173-mm long section a UCN shutter valve is located. The valve is made from a $0.5-\mathrm{mm}$ thick sheet of stainless steel with a hole (diameter $23 \mathrm{~mm}$ ) which can be rotated to positions between closing and fully opening the guide, via a rotary feedthrough to room temperature. A short conical section with a half opening angle of $75^{\circ}$ makes a transition to an 8-mm long straight section with inner diameter $50 \mathrm{~mm}$, followed by a $90^{\circ}$ bend with radius $70 \mathrm{~mm}$ connected with a horizontal, straight section of $342 \mathrm{~mm}$ length. A $65-\mathrm{mm}$ long conical section increases the inner guide diameter from 50 to $66 \mathrm{~mm}$. After an additional horizontal, 90- $\mathrm{mm}$ long straight section followed by a bend downwards with radius $80 \mathrm{~mm}$ a vertical, straight section with inner diameter $66 \mathrm{~mm}$ and length $1.65 \mathrm{~m}$ is connected. At the end of this section, the entrance foil to a UCN detector is situated. This foil with thickness $100 \mu \mathrm{m}$ is made from pure aluminum, followed by a 5-cm thick detection volume filled with 14 mbar of the strongly neutron absorbing gas ${ }^{3} \mathrm{He}, 1.1$ bar of $\mathrm{Ar}$, and 25 mbar of $\mathrm{CO}_{2}$ as quench gas [64]. The neutron detector is expected to have an efficiency close to $100 \%$ for UCN which enter the gas detector volume.

The cool-down of the apparatus including filling of the converter takes approximately one week and is presented in a time plot of four thermometers in Fig. 4. About 3 days after the cryocooler was started the converter vessel as well as the $1-\mathrm{K}$ and ${ }^{3} \mathrm{He}$ pots have thermalized below $10 \mathrm{~K}$. During the whole time helium is circulated in the two closed gas circuits to establish a good thermal contact and fast cooling. Afterwards, the ${ }^{4} \mathrm{He}$ is filled in the converter vessel via the superleak (approximately $1 \mathrm{~kg}$, visible in the plot as a period when the temperatures of the ${ }^{3} \mathrm{He}$ pot and the converter vessel are equal). This takes approximately another 2-3 days, before it is cooled from about $2 \mathrm{~K}$ to below $1 \mathrm{~K}$ within a few hours with the cooling power of the ${ }^{3} \mathrm{He}$ pot.

\section{CHARACTERIZATION OF THE BEAM LINE H172A}

The general outline of the new beam line has already been described in Ref. [31]. In the following section, we present its performance in detail.

\section{A. Neutron guide system and monochromator}

The superthermal UCN conversion mechanism in superfluid ${ }^{4} \mathrm{He}$ is most efficient within a narrow neutron wavelength band at $0.89 \mathrm{~nm}$ [48]. Placing the UCN source in a monochromatic neutron beam reduces the created $\gamma$ radiation and activation with respect to a converter situated in a white beam. In this case, multiphonon processes will not contribute to UCN production [46,47]. A top-view drawing of the beam line is depicted in Fig. 5. Neutrons from the white beam of neutron guide H172 coming from the vertical cold source of the ILL high-flux reactor are deflected by $\vartheta=61.2^{\circ}$ by means of a monochromator. They are fed into a secondary 4.5-m long neutron guide (H172a; items 3 and 5 in Fig. 5) equipped with a $m=2$ supermirror coating. ${ }^{2}$ The secondary neutron guide is tapered to reduce the cross section from $80 \times 80 \mathrm{~mm}^{2}$ to $70 \times 70 \mathrm{~mm}^{2}$ matching the cross section of the $\mathrm{BeO}$ ceramic pieces of the UCN storage volume.

The cold neutron beam incident on the UCN converter vessel can be switched on or off by means of a beam shutter, consisting of a 187-mm long block of lead, covered with $13 \mathrm{~mm}$ of $\mathrm{B}_{4} \mathrm{C}$, with a switching time of about $1 \mathrm{~s}$ (item 4 in Fig. 5). When opening the shutter, a piece of neutron guide integrated into the block is moved into the beam which avoids losses from beam divergence.

The monochromator is composed of 18 potassium intercalated graphite crystals of dimensions $45 \times 20 \times 3 \mathrm{~mm}^{3}$. Such crystals can be manufactured, depending on production conditions, to possess a structure with layers of potassium atoms situated between each layer of carbon atoms ("stage1 "-type crystal, with a lattice spacing $d_{1}=0.535 \mathrm{~nm}$ ) or between each second carbon layer ("stage-2" type crystal, $d_{2}=0.875 \mathrm{~nm}$ ). The beam line described here employs stage2 crystals to scatter a 0.89 -nm neutron beam by a first-order Bragg reflection under the angle $\vartheta$. The crystals have a peak reflectivity of $60 \%-70 \%$ and a mosaicity of about $2^{\circ}$, which matches the divergence of the incident beam [55]. They are

\footnotetext{
2 " $m=2$ " stands for a coating which provides a critical angle of total reflection two times larger than for nickel with natural isotopic composition.
}

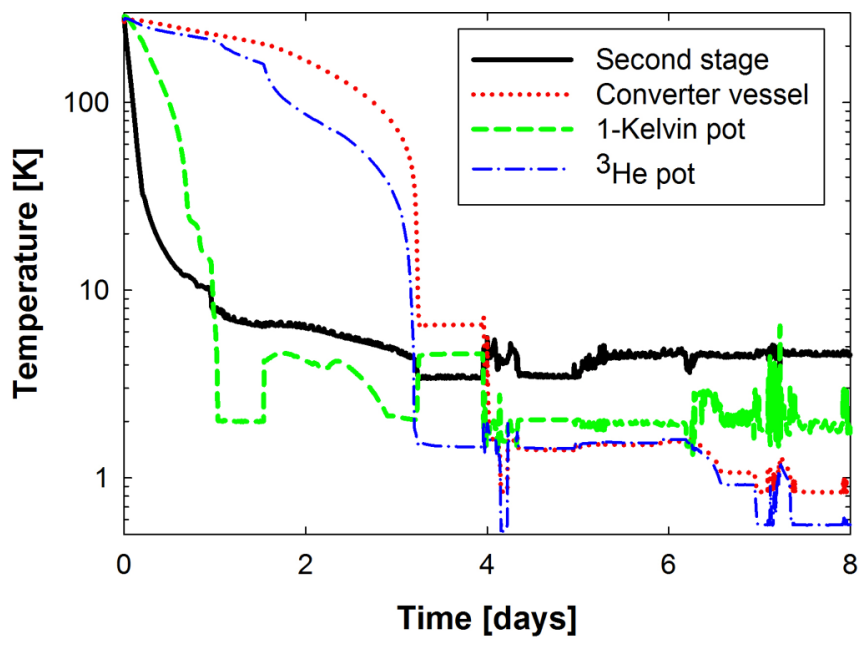

FIG. 4. (Color online) Time plot of a cool-down of the UCN source cryostat starting from room temperature. After about 7 days the filled converter reaches a temperature of $0.85 \mathrm{~K}$ (measured outside the converter at the UCN extraction side; see text), while the temperature in the ${ }^{3} \mathrm{He}$ pot is $0.55 \mathrm{~K}$. 


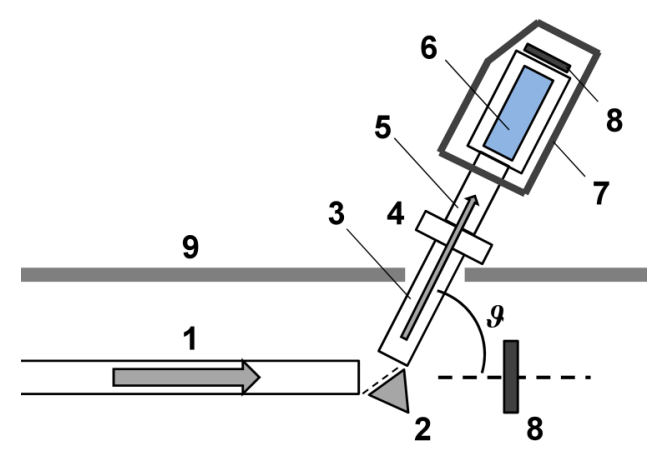

FIG. 5. (Color online) Schematic top view of the beam line: (1) neutron guide H172 coming from the vertical cold source of the ILL reactor, (2) monochromator crystals (dashed lines) attached to triangular shaped holder, (3) long neutron guide section of $\mathrm{H} 172 \mathrm{a}$, converging from $80 \times 80 \mathrm{~mm}^{2}$ to $70 \times 70 \mathrm{~mm}^{2}$ and tilted by $\vartheta=61.2^{\circ}$ with respect to the incoming neutron beam, (4) secondary beam shutter, (5) short neutron guide section of H172a, (6) UCN source with helium filled converter vessel, (7) lead shielding with a thickness of $7 \mathrm{~cm},(8)$ beam stops, and (9) concrete wall for biological shielding.

mounted with a gap of approximately $2 \mathrm{~mm}$ between two crystals, resulting in an estimated $90 \%$ coverage of the beam. The neutron flux on the monochromator is calculated to be $5.4 \times 10^{9} \mathrm{~cm}^{-2} \mathrm{~s}^{-1} \mathrm{~nm}^{-1}$ at $0.89 \mathrm{~nm}$ (at a reactor power of 58.3 MW). This value is based on a transmission simulation of the neutron guide, a gold foil activation measurement in front of the monochromator and spectral measurements made at the end of the neutron guide H171 [52]. ${ }^{3}$ Hence, assuming an integral neutron reflectivity of $40 \%$ and the coverage of the monochromator one expects a neutron flux of $2.0 \times 10^{9} \mathrm{~cm}^{-2} \mathrm{~s}^{-1} \mathrm{~nm}^{-1}\left(1.8 \times 10^{9} \mathrm{~cm}^{-2} \mathrm{~s}^{-1} \mathrm{~nm}^{-1}\right)$ at 0.89 $\mathrm{nm}$ and a reactor power of 58.3 MW (53.2 MW) entering the converter vessel [31]. ${ }^{4}$

\section{B. Neutron wavelength spectrum and beam image}

We evaluated the flux of the beam at the exit of the secondary guide by performing a gold foil activation analysis using a set of 13 gold foils equally distributed over the beam cross section. For the thermal equivalent flux we determined a mean value of $7.2 \times 10^{8} \mathrm{~cm}^{-2} \mathrm{~s}^{-1}$ (at a reactor power of 53.2 MW) and the beam intensity was homogeneous over the whole cross section within the precision of the method. To quantify the flux at $0.89 \mathrm{~nm}$, the wavelength distribution was measured in the center of the beam using a time-of-flight (TOF) setup. Its intrinsic resolution was about $\Delta \lambda=0.01 \mathrm{~nm}$ because of the opening time of the chopper and the detector thickness $(1 \mathrm{~cm})$. A correction because of neutron scattering and absorption in air was determined experimentally by comparison of

\footnotetext{
${ }^{3}$ The initial neutron guide $\mathrm{H} 17$ is split vertically into the guides H171 (reflectometer beam line Figaro) and H172 (beam line for UCN production).

${ }^{4}$ The integral reflectivity is lower than the stated value of $50 \%$ given in Ref. [31], because the peak reflectivity of the used crystals was found to be smaller $(60 \%-70 \%$ instead of $80 \%)$.
}

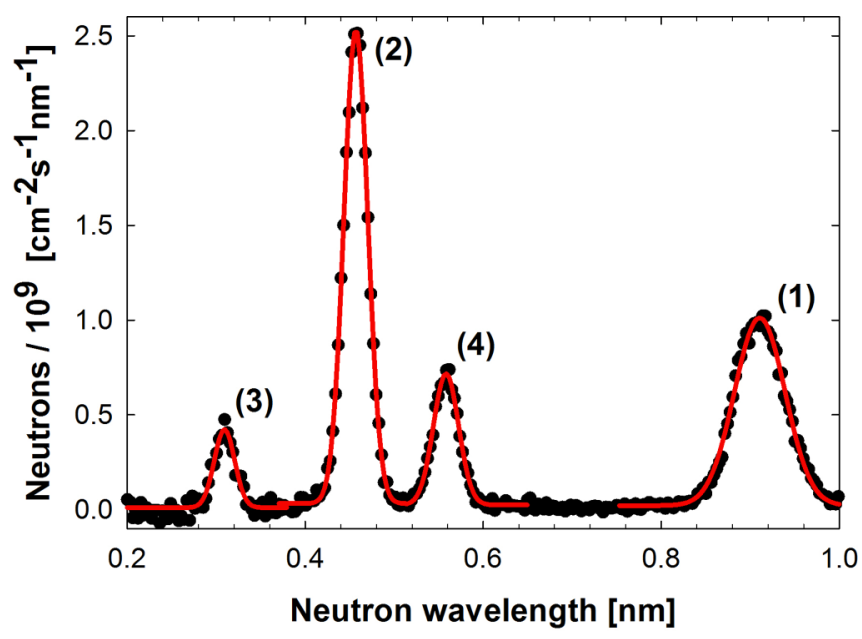

FIG. 6. (Color online) Time-of-flight spectrum taken at the exit of the secondary neutron guide in the center of the beam (corrected for neutron scattering and absorption in air and normalized to a reactor power of 53.2 MW). The positions and widths of the four peaks have been fitted with independent Gauss functions (red lines): $\lambda_{1}=(0.91 \pm 0.02) \mathrm{nm}, \sigma_{1}=0.0284(9) \mathrm{nm}, \lambda_{2}=(0.46 \pm 0.01) \mathrm{nm}$, $\sigma_{2}=0.0134(4) \mathrm{nm}, \lambda_{3}=(0.31 \pm 0.01) \mathrm{nm}, \sigma_{3}=0.0114(9) \mathrm{nm}$ and $\lambda_{4}=(0.56 \pm 0.01) \mathrm{nm}, \sigma_{4}=0.0142(5) \mathrm{nm}$. The given errors for the peak positions are dominated by the $2 \%$ uncertainty in the wavelength calibration (compare text). The errors on the peak width are a combination of statistical uncertainties and the wavelength calibration.

two TOF spectra measured with the secondary guide (items 3 and 4 in Fig. 5) vented with air and evacuated, respectively. The obtained wavelength dependent attenuation is about $15 \%$ $(11 \%)$ per meter at $0.91 \mathrm{~nm}(0.46 \mathrm{~nm})$. The corrected spectrum was normalized to the measured thermal equivalence flux and converted to the particle flux spectrum. The result is presented in Fig. 6. The plot shows four peaks: three of them $\left(\lambda_{1}\right.$, $\lambda_{2}$, and $\lambda_{3}$ ) correspond to the first three orders of Bragg reflections from scattering on stage- 2 type crystals and one peak $\left(\lambda_{4}\right)$ is caused by stage- 1 contamination in the crystals. The second-order peak $\left(\lambda_{2}\right)$ exceeds the first-order peak $\left(\lambda_{1}\right)$ because of the shape of the cold neutron spectrum incident on the monochromator exhibiting a broad maximum around $0.4 \mathrm{~nm}$ [52]. This measurement was performed with a short chopper to detector distance to assure that the full divergence of the different beam components is taken into account (the TOF setup was replacing the short neutron guide section; item 5 in Fig. 5). To correct for position and timing offsets two TOF spectra were taken at distances of about 425 and $675 \mathrm{~mm}$, each with an estimated systematic error of 2-3 $\mathrm{mm}$. This error results in an approximate $2 \%$ uncertainty in the calibration of the wavelength axis. The determined peak position at $\lambda_{1}=(0.91 \pm 0.02) \mathrm{nm}$, thus, agrees with the expected value of $0.89 \mathrm{~nm}$ within the given error. However, the spectrum also reveals a lack of intensity in the $\lambda_{1}$ peak with respect to the expected value presented previously. Taking into account the broadening due to the intrinsic TOF resolution, one determines a flux of $(1.0 \pm 0.2) \times 10^{9} \mathrm{~cm}^{-2} \mathrm{~s}^{-1} \mathrm{~nm}^{-1}$ at $0.89 \mathrm{~nm}$ and a reactor power of $53.2 \mathrm{MW}$. A $20 \%$ uncertainty, quoted on this 


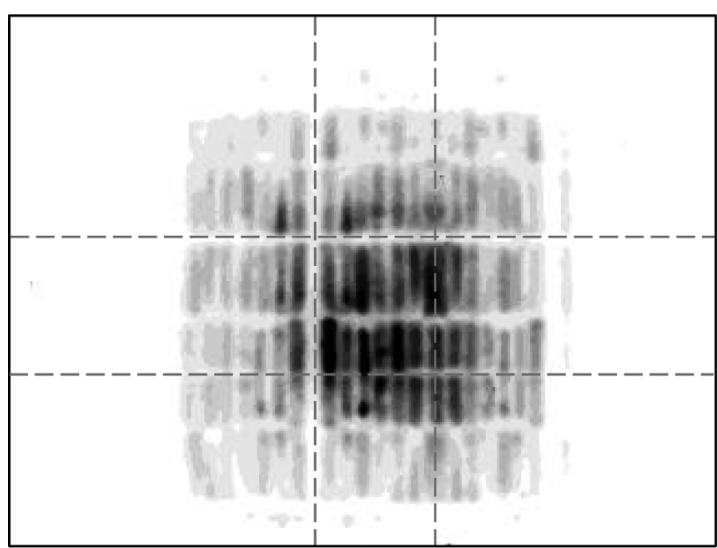

FIG. 7. Pinhole image of the beam of neutron guide H172a $(320 \times 240$ pixel). Dark (light) pixel correspond to a high (low) neutron flux intensity, i.e., a high (low) reflectivity of the crystals. The dashed lines indicate the central square which represents the image of the direct beam. The eight surrounding images are from mirror reflections in the secondary neutron guide.

result, was assigned to account for possible small deviations of the apparent peak position from $0.89 \mathrm{~nm}$ and uncertainties in the gold foil activation measurement. Normalized to equal reactor power, the differential flux is a factor 1.8 lower than expected. As the reduced flux could be caused by a nonperfect alignment of the crystals, a pinhole image of the beam was taken using a standard neutron CCD camera [56]. The result is presented in Fig. 7, showing that the individual crystals can be easily identified because of the gaps between them. Observable is also a variation of the crystal quality (and/or alignment, which cannot be judged from a single pinhole picture). Furthermore, the entire crystal stack seems to be mounted several millimeters too low, resulting in a white stripe with low intensity at the top of the direct beam image. Hence, it appears likely that part of the missing intensity can be recovered by optimizing the alignment and reflectivity of the monochromator crystals, and by reducing the gaps between them.

\section{PRODUCTION OF ULTRACOLD NEUTRONS}

A photograph of the UCN source is presented in Fig. 8. The source was characterized by performing several measurements which are described in this section. In these measurements the UCN are counted using a ${ }^{3} \mathrm{He}$ gas detector (with an efficiency close to $100 \%$ ). It is attached to the extraction guide made from stainless steel with a bend downward to let the UCN gain gravitational energy to increase the transmission probability through the aluminum entrance window of the detector (neutron optical potential of $54 \mathrm{neV}$ ), as shown in Fig. 1 and visible on the right in Fig. 8.

\section{A. UCN production as a function of temperature}

The cooldown of the superfluid ${ }^{4} \mathrm{He}$ from $1.5 \mathrm{~K}$ to the base temperature of $0.7 \mathrm{~K}$ was slow enough to follow the increase of the UCN density in the storage volume as a function of temperature. For that purpose the UCN shutter valve was

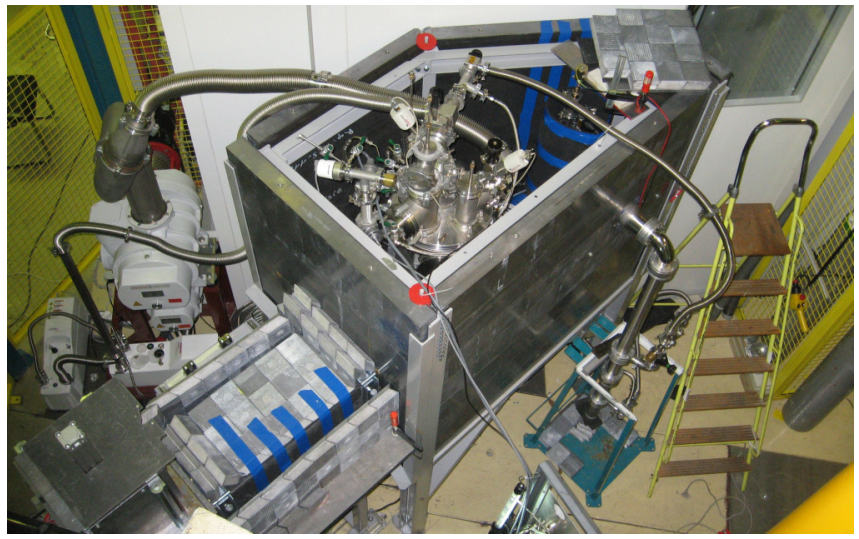

FIG. 8. (Color online) Setup of the new UCN source at the beam line H172a. The cryostat containing the superfluid helium converter is housed in a lead castle. Shown is the setup with sidewards UCN extraction as used in the experiments described here. The shutter for the cold neutron beam is situated in the lower left corner of the picture.

slightly opened providing a small slit for the UCN to leak from the production volume to the detector. Figure 9(a) depicts the buildup and decay of the UCN leakage rate measured at four different temperatures as a function of time after opening and closing the cold neutron shutter, i.e., irradiation of the converter vessel with cold neutrons. The rise of the count rates to a plateau is well described by a single exponential buildup. The fit parameters "saturated UCN leakage rate" and "buildup time constant" $\tau_{\mathrm{b}}$ are presented as a function of temperature $T$ in Fig. 9(b). The plot demonstrates the expected proportionality between these two parameters. Compared to a separate measurement, where the buildup time constant with a completely closed shutter valve was determined to be $(67 \pm 3)$ s at $0.7 \mathrm{~K}$ (compare below), here, the buildup time reaches a saturation value of $46 \mathrm{~s}$. This indicates that the storage lifetime of the UCN in the storage volume is limited by the slightly opened shutter valve.

The temperature dependence of the buildup time constant was fitted using the function,

$$
\frac{1}{\tau_{\mathrm{b}}(T)}=\frac{1}{\tau_{0}}+\frac{1}{\tau^{\prime}} \cdot T^{c},
$$

where $T$ is the ${ }^{4} \mathrm{He}$ temperature in units of Kelvin and with the fit parameters $\tau_{0}, \tau^{\prime}$ (time constants), and $c$. A least-squares fit to the data points yields $\tau_{0}=(46 \pm 1) \mathrm{s}, \tau^{\prime}=(111 \pm 7) \mathrm{s}$, and $c=7.2 \pm 0.3$. Here, $\tau_{0}$ is specific for our UCN storage volume with the slightly opened UCN shutter valve, while the storage time constant in ${ }^{4} \mathrm{He}$ at $1 \mathrm{~K}, \tau^{\prime}$, and the exponent $c$ generally describe the temperature-dependent up-scattering of $\mathrm{UCN}$ in liquid ${ }^{4} \mathrm{He}$. The obtained values are in agreement with the findings given in Refs. [57,58] and with the two-phonon up-scattering term derived in Ref. [59]. The corresponding fit curve is presented in Fig. 9(b). In a measurement with the fully opened UCN valve performed at $1.27 \mathrm{~K}$ we obtained a continuous UCN rate of $1400 \mathrm{~s}^{-1}$ through the extraction hole with area $4 \mathrm{~cm}^{2}$. 

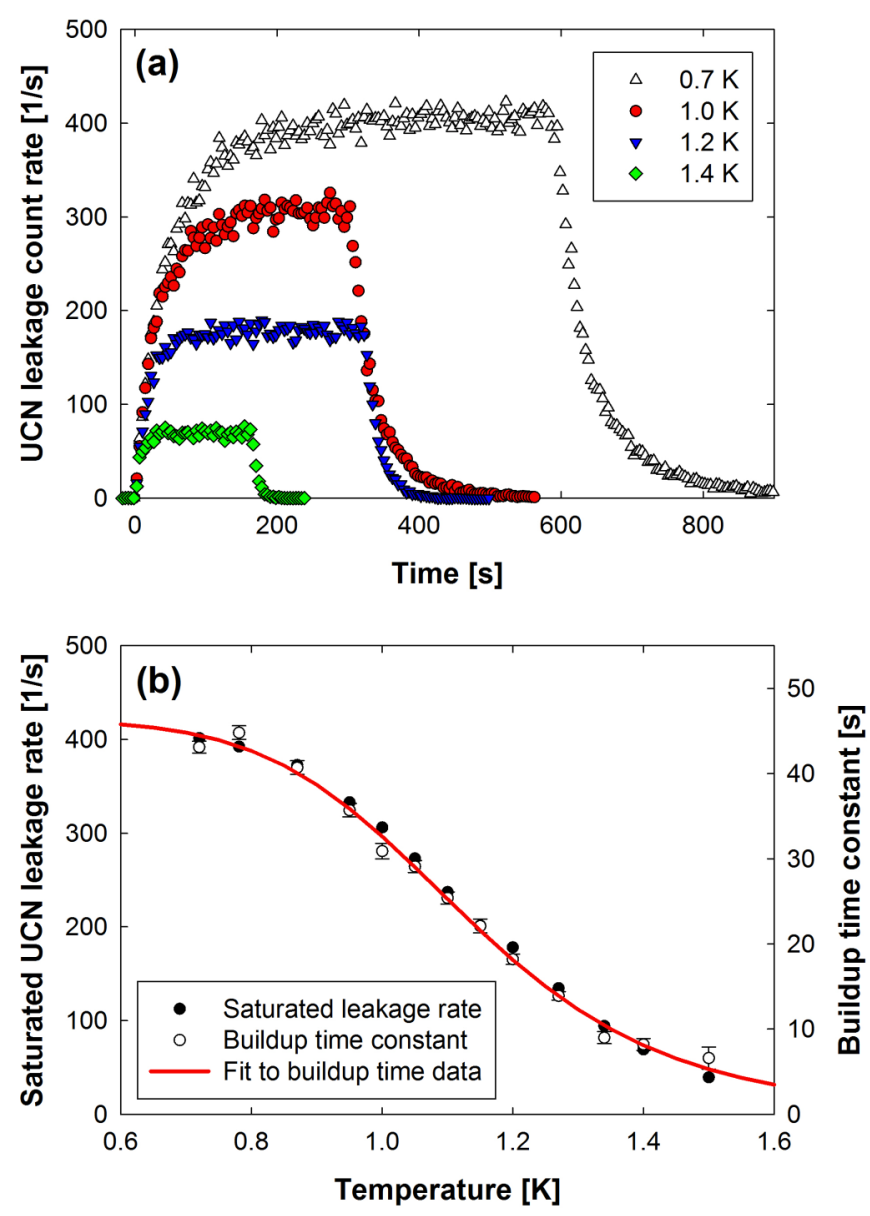

FIG. 9. (Color online) (a) UCN leakage rate from the UCN storage volume to the detector with a slightly opened UCN shutter valve for different temperatures of the superfluid ${ }^{4} \mathrm{He}$. The converter vessel is irradiated with cold neutrons for different durations with starting time set to zero. For clarity 4 neighboring data points have been averaged and recombined to one. (b) Saturated UCN leakage rate through the slightly opened shutter valve and the corresponding buildup time constant for different ${ }^{4} \mathrm{He}$ temperatures. The latter are obtained from a fit to the exponential rise of the data points in (a). The line represents a fit to the data points of the buildup time (compare text).

\section{B. Storage lifetime measurements and UCN density}

The storage lifetime of the UCN in the production volume is limited by several loss mechanisms: (i) the neutron $\beta$ decay with $\tau_{\beta} \approx 880 \mathrm{~s}$, (ii) phonon up-scattering processes in liquid ${ }^{4} \mathrm{He}$, (iii) absorption by a residual ${ }^{3} \mathrm{He}$ contamination in the liquid helium, (iv) absorption and up-scattering from wall collisions of the neutrons, (v) absorption and up-scattering by impurities, and (vi) losses through gaps in the storage volume wall.

As determined above the effect from the up-scattering in ${ }^{4} \mathrm{He}$ can be approximated at low temperatures by $\tau_{\text {up }} \approx$ $110 \mathrm{~s} / T^{7}$, where $T$ is in units of Kelvin. For $0.7 \mathrm{~K}$ this yields about 1350 s. Neutron losses from wall collisions depend on the neutron velocity and the mean free path given by $\bar{l}=4 V / A$, where $A$ and $V$ are the total surface area and volume of the storage volume (here $A \approx 0.3 \mathrm{~m}^{2}, V \approx 5$ liter, and $\bar{l} \approx 7 \mathrm{~cm}$ ). For estimates, we employ the maximum neutron velocity $v_{\max }$ and the maximum loss probability per wall collision $\mu_{\max } \equiv \pi f$, with $f$ being the so-called UCN loss factor (for $\mathrm{BeO}, \mu_{\max } \approx 4 \times 10^{-5}$ ) [4]. From the kinetic UCN gas theory it follows that $\tau_{\text {wall }}=\bar{l} /\left(v_{\max } \mu_{\max }\right)$. Hence, for the maximal UCN velocity of $6 \mathrm{~m} / \mathrm{s}$, limited by the neutron optical potential of the vertical stainless steel guide, this yields $\tau_{\text {wall }} \approx 300 \mathrm{~s}$. Taking only these two loss mechanisms and the $\beta$ decay into account, one obtains an estimated upper limit for the storage lifetime in our UCN production volume of $\tau^{*} \approx 200 \mathrm{~s}$.

To determine the storage lifetime and time constants for the UCN extraction, we performed delayed UCN extraction experiments. In these measurements the converter vessel is irradiated with cold neutrons for a period much longer than the buildup time constant, while the UCN shutter valve remains closed. The UCN valve is then opened with a delay time after closing the beam. In Fig. 10(a) typical time plots of the UCN count rate are shown for the superfluid ${ }^{4} \mathrm{He}$ held at $0.7 \mathrm{~K}$. During the 300-s long irradiation with cold neutrons the buildup of UCN density can be followed because of the leaking of UCN through the fully closed shutter valve. After closing the beam the density decays with a time constant in agreement with the buildup time constant. An exponential fit to the data points yields $(67 \pm 3) \mathrm{s}$ for the buildup and $(70 \pm 2) \mathrm{s}$ for the decay time constant. After the delayed opening of the shutter valve the accumulated UCN are emptied from the source to the detector. The integral UCN counts in such an emptying curve are presented in Fig. 10(b) as a function of the delay time. Here, the integral counts are summed over the first $60 \mathrm{~s}$ after opening the shutter valve. A double exponential fit to the data points yields two time constants: $\tau_{1}=(43 \pm 1) \mathrm{s}$ and $\tau_{2}=(132 \pm 2) \mathrm{s}$, with approximately $78 \%$ of the measured intensity in the faster component. The presence of somewhat different time constants is characteristic for a broad UCN energy spectrum stored in a material bottle because the frequency of wall collisions and the average loss probability per wall collision are strongly energy dependent. The resulting effect of spectral shaping of the trapped UCN is well visible in the impressive rise of extraction time constants with increasing delay time presented in Fig. 10(c). ${ }^{5}$

On the other hand, the UCN absorption by residual ${ }^{3} \mathrm{He}$ in the liquid helium does not depend on the neutron velocity and, thus, we can provide an upper limit for the isotopical purity of ${ }^{3} \mathrm{He} /{ }^{4} \mathrm{He}<10^{-10}$. This limit is obtained by attributing the full difference between the estimated storage lifetime $\tau^{*}$ and the measured longer time constant $\tau_{2}$ to the ${ }^{3} \mathrm{He}$ contamination via $1 / \tau^{3} \mathrm{He}=1 / \tau_{2}-1 / \tau^{*} \approx(400 \mathrm{~s})^{-1}$, and employing the thermal neutron absorption cross section of ${ }^{3} \mathrm{He}$ of 5330 barn. $^{6}$

\footnotetext{
${ }^{5}$ Note that for long delay times because of the longer extraction time constants, the summation of neutron counts over the first $60 \mathrm{~s}$ after opening the UCN valve yields an underestimation of the neutron count rate and consequently of $\tau_{2}$.

${ }^{6} \mathrm{The}{ }^{3} \mathrm{He}$ number density can be estimated by $\rho_{3} \mathrm{He}=\left(\tau_{3} \mathrm{He}\right.$. 5330 barn $\cdot 2200 \mathrm{~m} / \mathrm{s})^{-1}$.
} 

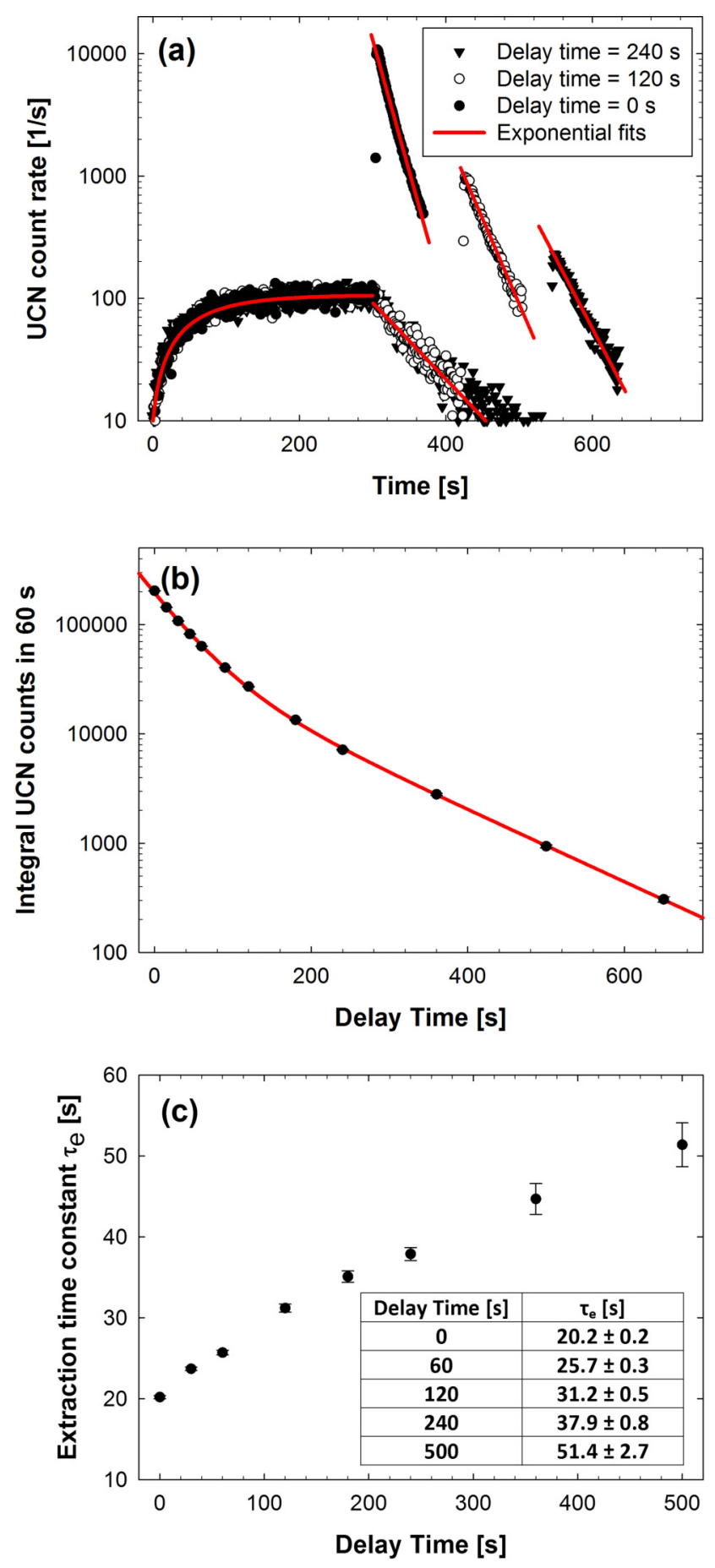

FIG. 10. (Color online) (a) Time plots of the delayed extraction measurements at a ${ }^{4} \mathrm{He}$ temperature of about $0.7 \mathrm{~K}$. The plot depicts the detected UCN count rate as a function of time and corresponding single exponential fit curves (red lines). The converter vessel is irradiated with the cold neutron beam for a 300-s long UCN accumulation period. After the irradiation and a variable delay time the UCN shutter valve is opened and the UCN are extracted towards the detector. (b) The integral counts of the extracted $\mathrm{UCN}$ as a function of the delay time. The line shows the fit with a double exponential decay function. (c) Extraction time constant $\tau_{\mathrm{e}}$ as a function of the delay time, obtained from a single exponential decay fit to the data points in (a) during emptying of the storage volume.

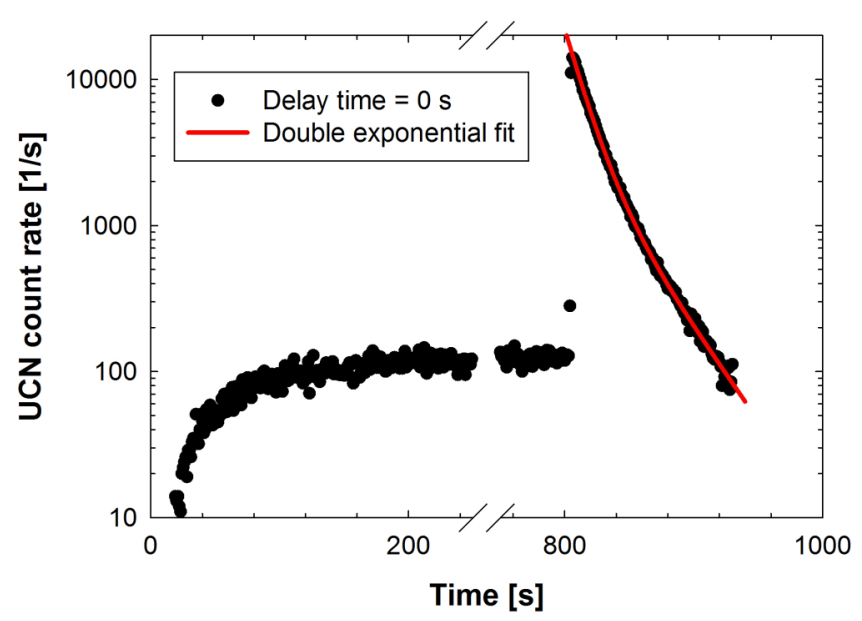

FIG. 11. (Color online) Time plot of a delayed extraction measurement with zero delay time. Here, the UCN shutter valve is opened after approximately $800 \mathrm{~s}$ of irradiation with cold neutrons and accumulation of UCN. The total number of detected neutrons during the 125 -s long extraction is approximately $2.74 \times 10^{5}$. The double exponential fit to the extraction data yields two time constants: $(13 \pm 1) \mathrm{s}$ and $(34 \pm 1) \mathrm{s}$.

In conclusion, the experimentally determined buildup and decay time constants are about a factor 3 smaller than $\tau^{*}$. This can be attributed to losses through gaps, for instance, between the individual $\mathrm{BeO}$ ceramic pieces (e.g., a relatively large combined slit area of $0.3 \mathrm{~cm}^{2}$ would lead to an additional loss channel with a time constant of about $100 \mathrm{~s}$ ), and losses from absorption or up-scattering by possible impurity depositions on the walls of the production volume.

An extraction measurement with a longer emptying period is presented in Fig. 11. From this a lower limit for the UCN density in the production volume can be determined, not taking losses from an imperfect UCN guide system into account. In this measurement a total number of $2.74 \times 10^{5} \mathrm{UCN}$ was counted, corresponding to a density of at least $55 \mathrm{~cm}^{-3}$.

\section{Duty cycle of the UCN source}

In the presented experiments, we observed no significant heating of the source from the irradiation with the neutron beam. On the other hand, after opening the UCN shutter valve the temperature of the superfluid ${ }^{4} \mathrm{He}$ increases because of thermal radiation entering the bath along the extraction guide. However, as most UCN experiments involve filling experimental bottles in a periodic manner (e.g., neutron lifetime and electric dipole moment experiments), the valve needs to be open only for short moments between much longer periods of manipulation or storage of the extracted UCN. The UCN density in the source can be replenished during these periods. To study a typical operation of the source we repeated UCN extractions with the neutron beam switched on all the time. In Fig. 12 measured UCN counts for a duty cycle of $10 \%$ are shown, where in each cycle the shutter valve is kept open for $10 \mathrm{~s}$, followed by a UCN accumulation time of $90 \mathrm{~s}$. The total number of counts in each emptying curve was approximately $1.4 \times 10^{5}$. Within $1 \mathrm{~h}$ of operation the ${ }^{4} \mathrm{He}$ temperature rose 


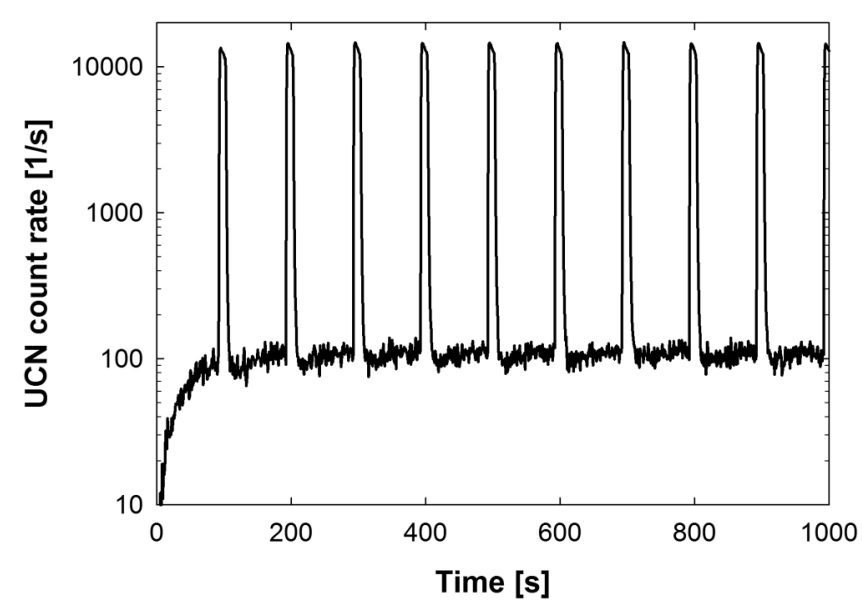

FIG. 12. Repeated extraction of UCN during permanent irradiation of cold neutrons with a duty cycle of $10 \%$ (defined as fraction of time with UCN shutter valve open per accumulation-extraction cycle). In each emptying curve a total amount of approximately $1.4 \times 10^{5}$ $\mathrm{UCN}$ is extracted (shutter valve open for $10 \mathrm{~s}$, counting during $20 \mathrm{~s}$ after start of shutter opening).

from 0.7 to $0.9 \mathrm{~K}$, associated with a small drop in UCN count rate. Hence, without provision of additional cooling power, a slightly reduced duty cycle should be applied to maintain a constant UCN output. For a duty cycle of 5\% the mean temperature of the helium bath stayed at $0.7 \mathrm{~K}$ without any observable drift [53].

\section{Comparison with expected UCN density}

For an estimate of the UCN density $\rho_{\mathrm{UCN}}$ to be expected for this source, we neglect energy dependencies and write $\rho_{\mathrm{UCN}}=p \tau$ with an ensemble average value for $\tau$, for which we use $(67 \pm 3) \mathrm{s}$ as determined from the UCN buildup measurements through the leaking $\mathrm{UCN}$ valve. The production rate density $p=\int_{0}^{U} p(\epsilon) d \epsilon$ is defined as the conversion rate of cold neutrons to UCN with kinetic energies smaller than the trap potential depth $U$ (accounting for the neutron optical potential of superfluid ${ }^{4} \mathrm{He}$ of $19 \mathrm{neV}$ ). For our converter vessel, the wall material with the smallest neutron optical potential is uncoated stainless steel of the vertical UCN guide section and the shutter valve (items 17 and 18 in Fig. 1). Hence, from $p \propto U^{3 / 2}$ and using the value calculated for a vessel made of beryllium [47] one derives $p=(3.0 \pm 0.2) \times$ $10^{-9} d \phi /\left.d \lambda\right|_{\lambda^{*}} \mathrm{~cm}^{-3} \mathrm{~s}^{-1}$, with the differential neutron flux at $\lambda^{*}=0.89 \mathrm{~nm}$ given in $\mathrm{cm}^{-2} \mathrm{~s}^{-1} \mathrm{~nm}^{-1}$. Because the vessel's side walls from sintered $\mathrm{BeO}$ ceramics do not guide the cold neutron beam, this value needs to be divided by a factor of $1.4 \pm 0.1$ to obtain the spatially averaged production rate density, where the stated value is based on simple geometrical considerations. For the neutron flux as obtained from the TOF measurements, we thus expect $\rho_{\mathrm{UCN}} \approx(140 \pm 20) \mathrm{cm}^{-3}$. On the other hand, the experimental value of $55 \mathrm{~cm}^{-3}$ quoted before was exclusively based on detected UCN. For a valid comparison one needs to apply a correction by the ratio $\left(\tau+\tau_{\mathrm{e}}\right) / \tau \approx 1.3$ to account for UCN losses within the converter during extraction with an extraction time constant of $\tau_{\mathrm{e}} \approx 20$ s, i.e., $\rho_{\mathrm{UCN}}^{\exp } \approx 70 \mathrm{~cm}^{-3}$. The agreement looks not unreasonable because we did not take into account the transmission of the UCN extraction guide with its two $90^{\circ}$ bends, the transmission of the detector entrance window and apertures for pumping.

\section{CONCLUSION AND OUTLOOK}

The installation of the apparatus described here represents a milestone towards a "next generation" UCN source. The measured UCN density exceeds that of the longstanding user source PF2 at ILL. However, there is still a lot of room for improvements, and it is instructive to consider identified factors $f_{i}$ that would result in a significant gain in $\mathrm{UCN}$ density if the following measures are taken. A first gain will result from a coating of all parts inside the production volume and of the UCN guides with beryllium or any other material with high neutron optical potential and low absorption, such as diamondlike carbon [60-62] or cubic ${ }^{11} \mathrm{BN}$ [63]. For beryllium the increased trap depth with respect to the vessel partly made of stainless steel, will yield a gain factor $f_{1}=1.67$. Second, for the given geometry, an effective storage time constant of approximately $200 \mathrm{~s}$ should be feasible (compare $\left.\tau^{*}\right){ }^{7}$ The improved storage lifetime would correspond to a gain factor $f_{2}=3$. Third, the mentioned loss from beam divergence can be avoided if the side walls of the converter vessel guide the cold neutron beam, i.e., $f_{3}=1.4 \pm 0.1$. This can be achieved by sputtering a supermirror coating on the production volume walls before depositing the UCN reflecting top layer. Neglecting any further gain from improved UCN extraction efficiency, the saturated UCN density in the source installed at its present beam location H172a with characteristics as described in this paper would thus become $f_{1} f_{2} f_{3} \cdot 70 \mathrm{~cm}^{-3}=(490 \pm 40) \mathrm{cm}^{-3}$.

In the meantime a new secondary beam position, H172b, with similar characteristics was put in operation. This beam line will be used to bring the technique of superthermal UCN production in superfluid ${ }^{4} \mathrm{He}$ to maturity using a successor of the UCN source cryostat presented here. Because the monochromation losses were found to be significantly larger than initially anticipated, another large gain could be achieved by omission of the monochromator and implementation of the source in the direct/white beam at the exit of the guide H172, where the differential neutron flux at $0.89 \mathrm{~nm}$ is about a factor 5 higher than in the secondary beam [52].

\section{ACKNOWLEDGMENT}

The authors gratefully acknowledge help and support from D. Berruyer, B. van den Brandt, T. Brenner, M. Guigou, S. Mironov, and the entire GRANIT collaboration.

\footnotetext{
${ }^{7}$ Note that the Cryo-EDM collaboration, with a superthermal source consisting of a $3 \mathrm{~m}$ long tubular vessel with $63 \mathrm{~mm}$ diameter made of beryllium-coated copper and closed off by beryllium windows, but without extraction of UCN through a liquid helium-vacuum interface, has measured a storage time of $160 \mathrm{~s}$ [7].
} 
[1] D. Dubbers and M. G. Schmidt, Rev. Mod. Phys. 83, 111 (2011).

[2] M. J. Ramsey-Musolf and S. Su, Phys. Rept. 456, 1 (2008).

[3] H. Abele, Prog. Nucl. Phys. 60, 1 (2008).

[4] R. Golub, D. J. Richardson, and S. K. Lamoreaux, Ultra-Cold Neutrons (Taylor \& Francis, New York, 1991).

[5] C. A. Baker et al., Phys. Rev. Lett. 97, 131801 (2006).

[6] I. Altarev et al., Phys. Atom. Nucl. 59, 1152 (1996).

[7] M. G. D. van der Grinten, Nucl. Instr. Meth. A 611, 129 (2009).

[8] Y. Masuda, K. Asahi, K. Hatanaka et al., Phys. Lett. A 376, 1347 (2012).

[9] T. M. Ito, J. Phys. Conf. Ser. 69, 012037 (2007).

[10] I. Altarev et al., Nuovo Cim. C035N04, 122 (2012).

[11] C. Baker, G. Ban, K. Bodek et al., Physics Procedia 17, 159 (2011).

[12] A. P. Serebrov et al., Nucl. Instr. Meth. A 611, 263 (2009).

[13] S. K. Lamoreaux and R. Golub, J. Phys. G: Nucl. Part. Phys. 36, 104002 (2009).

[14] G. Ban, K. Bodek, M. Daum et al., Phys. Rev. Lett. 99, 161603 (2007).

[15] A. P. Serebrov, E. B. Aleksandrov, N. A. Dovator et al., Phys. Lett. B 663, 181 (2008).

[16] I. Altarev, C. A. Baker, G. Ban et al., Phys. Rev. Lett. 103, 081602 (2009).

[17] I. Altarev et al., Eur. Phys. Lett. 92, 51001 (2010).

[18] S. Baeßler, V. V. Nesvizhevsky, K. V. Protasov, and A. Yu. Voronin, Phys. Rev. D 75, 075006 (2007).

[19] A. P. Serebrov, O. Zimmer, P. Geltenbort et al., JETP Lett. 91, 6 (2010).

[20] O. Zimmer, Phys. Lett. B 685, 38 (2010).

[21] A. Pichlmaier, V. Varlamov, K. Schreckenbach, and P. Geltenbort, Phys. Lett. B 693, 221 (2010).

[22] A. P. Serebrov, V. E. Varlamov, A. G. Kharitonov et al., Phys. Rev. C 78, 035505 (2008).

[23] S. S. Arzumanov et al., JETP Lett. 95, 224 (2012).

[24] P. R. Huffman et al., Nature (London) 403, 62 (2000).

[25] S. Paul, Nucl. Instr. Meth. A 611, 157 (2009).

[26] M. P. Mendenhall et al., Phys. Rev. C 87, 032501(R) (2013).

[27] V. V. Nesvizhevsky, H. G. Börner, A. K. Petukhov, H. Abele et al., Nature (London) 415, 297 (2002).

[28] T. Jenke, P. Geltenbort, H. Lemmel, and H. Abele, Nature Phys. 7, 468 (2011).

[29] T. Jenke et al., arXiv:1208.3875.

[30] M. Kreuz, V. V. Nesvizhevsky, P. Schmidt-Wellenburg et al., Nucl. Instr. Meth. A 611, 326 (2009).

[31] P. Schmidt-Wellenburg et al., Nucl. Instr. Meth. A 611, 267 (2009).

[32] A. Steyerl et al., Phys. Lett. A 116, 347 (1986).

[33] U. Trinks, F. J. Hartmann, S. Paul, and W. Schott, Nucl. Instr. Meth. A 440, 666 (2000).

[34] Y. Masuda, K. Hatanaka, S.-C. Jeong et al., Phys. Rev. Lett. 108, 134801 (2012).
[35] A. Saunders, M. Makela, Y. Bagdasaova et al., Rev. Sci. Instrum. 84, 013304 (2013).

[36] E. I. Korobkina, B. W. Wehring, A. I. Hawari, A. R. Young et al., Nucl. Instr. Meth. A 579, 530 (2007).

[37] A. Frei, Y. Sobolev, I. Altarev et al., Eur. Phys. J. A 34, 119 (2007).

[38] A. P. Serebrov, V. A. Mityuklaev, A. A. Zakharov et al., Nucl. Instr. Meth. A 611, 276 (2009).

[39] A. Anghel, F. Atchison, B. Blau et al., Nucl. Instr. Meth. A 611, 272 (2009).

[40] J. Karch et al., arXiv:1308.4610.

[41] T. Lauer and T. Zechlau, Eur. Phys. J. A 49, 104 (2013).

[42] R. Golub and J. M. Pendlebury, Phys. Lett. 53A, 133 (1975).

[43] R. Golub and J. Pendlebury, Phys. Lett. A 62, 337 (1977).

[44] J. Beringer et al., Phys. Rev. D 86, 010001 (2012).

[45] F. E. Wietfeldt and G. L. Greene, Rev. Mod. Phys. 83, 1173 (2011).

[46] E. Korobkina, R. Golub, B. W. Wehring, and A. R. Young, Phys. Lett. A 301, 462 (2002).

[47] P. Schmidt-Wellenburg, K. H. Andersen, and O. Zimmer, Nucl. Instr. Meth. A 611, 259 (2009).

[48] C. A. Baker, S. N. Balashov, J. Butterworth et al., Phys. Lett. A 308, 67 (2003).

[49] A. I. Kilvington et al., Phys. Lett. A 125, 416 (1987).

[50] O. Zimmer, K. Baumann, M. Fertl et al., Phys. Rev. Lett. 99, 104801 (2007).

[51] O. Zimmer, P. Schmidt-Wellenburg, M. Fertl et al., Eur. Phys. J. C 67, 589 (2010).

[52] K. Andersen (unpublished).

[53] O. Zimmer, F. M. Piegsa, and S. N. Ivanov, Phys. Rev. Lett. 107, 134801 (2011).

[54] P. Schmidt-Wellenburg and O. Zimmer, Cryogenics 46, 799 (2006).

[55] P. Courtois et al., Nucl. Instr. Meth. A 634, S37 (2011).

[56] Neutron Optics Grenoble, http://www.neutronoptics.com/ cameras.html.

[57] R. Golub, C. Jewell, P. Ageron, W. Mampe, B. Heckel, and I. Kilvington, Z. Phys. B 51, 187 (1983).

[58] H. Yoshiki et al., Phys. Rev. Lett. 68, 1323 (1992).

[59] R. Golub, Phys. Lett. A 72, 387 (1979).

[60] M. G. D. van der Grinten, J. M. Pendlebury, D. Shiers et al., Nucl. Instr. Meth. A 423, 421 (1999).

[61] F. Atchison, B. Blau, M. Daum et al., Phys. Rev. C 74, 055501 (2006)

[62] F. Atchison, B. Blau, M. Daum et al., Nucl. Instr. Meth. B 260, 647 (2007).

[63] Yu. Sobolev, Th. Lauer, Yu. Borisov et al., Nucl. Instr. Meth. A 614, 461 (2010).

[64] L. V. Groshev, V. N. Dvoretsky, A. M. Demidov, V. I. Luschikov, S. A. Nikolaev, Y. N. Panin, Y. N. Pokotilovsky, A. V. Strelkov, and F. L. Shapiro, JINR Dubna Commun. P3-7282 (1973). 